\title{
Laser surface texturing for antiviral surfaces?
}

\section{Nagarajan, Balasubramanian}

Department of Mechanical Engineering and Flanders Make@KU Leuven-MaPS, KU Leuven

Celestijnenlaan 300, 3001 Leuven, Belgium

balasubramanian.nagarajan@kuleuven.be

Yildirim, Kerim

Department of Mechanical Engineering and Flanders Make@KU Leuven-MaPS, KU Leuven

Celestijnenlaan 300, 3001 Leuven, Belgium

kerim.yildirim@kuleuven.be

\section{Saravanan, Rathi}

Lee Kong Chian School of Medicine, Nanyang Technological University Singapore

59 Nanyang Drive, Singapore 636921, Singapore

rathi@ntu.edu.sg

Castagne, Sylvie ${ }^{1}$

Department of Mechanical Engineering and Flanders Make@KU Leuven-MaPS, KU Leuven

Celestijnenlaan 300, 3001 Leuven, Belgium

sylvie.castagne@keleuven.be

\begin{abstract}
The coronavirus (COVID-19) pandemic, especially, the transmission of the virus via infected contact surfaces especially, has put increased emphasis on the need to fabricate antimicrobial surfaces to protect against various deadly pathogens. Laser surface texturing (LST), one of the common surface modification techniques, has been successful for antifouling applications to improve resistance against bacterial adhesion. In this short review, we aim to explore the possibilities of using LST for fabricating surfaces against viruses. The characteristics influencing the interaction of surfaces with virus and bacteria, and an overview of antibacterial surfaces created by LST are briefly described first before assessing the current limitations with LST for fabricating antiviral surfaces. Finally, we highlight the potential directions using LST to realize the nanoscale surface features necessary for antiviral surfaces.
\end{abstract}

${ }^{1}$ Corresponding author 


\section{Introduction}

Contact surfaces and contact surface disinfection has gained much attention with the recent coronavirus (COVID-19) pandemic [1]. Although inhalation of virus-laden respiratory droplets is the major route of transmission, the potential risk of contracting severe acute respiratory syndrome coronavirus-2 (SARS-CoV-2) via surface-mediated transmission is high considering the virus particles viability up to $72 \mathrm{~h}$ on stainless steel and plastic, $24 \mathrm{~h}$ on cardboard and $8 \mathrm{~h}$ on copper [1,2]. Similar studies with other viruses (SARS-CoV-1, MERS-CoV, human coronavirus 229E and influenza A virus) have shown that harmful viruses persist on surfaces and remain infectious for weeks [3]. These reports highlight the need for consistent disinfection of commonly accessible surfaces such as bed rails and door handles in hospitals and community settings, as a measure to contain the spread of the pandemic. However, common surface disinfection procedure is laborious and time-consuming. Multiple factors, such as material and environmental conditions influence the persistence of coronaviruses on surfaces [4] and thus there is an ultimate necessity to create durable contact surfaces with antimicrobial properties to reduce the surface-driven transmission of virus particles.

To this end, in this short review, we first present an overview of the physical and chemical characteristics influencing virus-surface adhesion, current fabrication techniques employed in developing antimicrobial surfaces with a special focus on laser surface texturing (LST), and its potential use in developing antiviral surfaces. Notably, the current literature on antimicrobial surfaces focuses primarily on bacteria, more common and larger contaminating pathogens than viruses [5]. Given this technological gap on the utilization of LST for the fabrication of antiviral surfaces, we attempt here to evaluate the possibility to apply the available knowledge on antibacterial surface texturing to antiviral surfaces based on the specific mechanisms of absorption and retention of microorganisms on metallic surfaces.

\section{Fabrication of antimicrobial surfaces}

\subsection{Antiviral surfaces}

Fabrication of antimicrobial surfaces to prevent the adhesion of microorganisms has been studied over the past few decades for many applications including biomedical devices and marine components [6,7]. Otter et al. [8] proposed the following criteria for the design of antimicrobial surfaces: safe for human contact, economically viable, simple processing technology, high longevity, rapid antimicrobial activity, compatible for regular cleaning as well as retention of microbial resistance despite the accumulation of dirt/organic matter on the surfaces with regular use. In order to achieve surfaces with desirable antiviral properties two essential characteristics need to be controlled: a) the adsorption of the virus to the surface, and b) viability of the virus. Firstly, the quantity of adsorbed virus is influenced by multiple factors including surface chemistry of the substrate, characteristics of the virus outer surface proteins such as surface electric charge, size, stability, and environmental conditions [9]. Electrostatic and van der Waals interactions are the two common mechanisms by which virus adsorbs to surfaces. Secondly, the persistence or the viability of the virus on the surfaces is influenced by several variables including humidity, temperature, and surface chemistry [4]. In the case of SARS-CoV-2, spike (S) glycoprotein, a major component protruding through the viral membrane, significantly influences the absorption of the virus to surfaces [10]. Temperature influences virus stability inversely, as an increase in temperature from $37^{\circ} \mathrm{C}$ to $70{ }^{\circ} \mathrm{C}$ reduced the virus lifetime from 1 day to 5 min [11]. Likewise, the high surface temperature is beneficial for better antiviral characteristics, due to reduced water bridging around the virus $[9,12]$. Studies conducted on similar coronaviruses such as SARS-CoV-1 and MERS-CoV indicated that high temperature and humidity generally reduce the viability of the virus particles [4]. Contrastingly, Joonaki et al. [9] highlight that high humidity increases the hydrogen bonding between the water molecules and the proteins at the outer surface of the virus, in addition to filling the gap between the virus particles.

Surface coatings, especially inorganic and polymer-based nanostructured coatings, are one of the most common strategies to achieve antiviral surface characteristics [13]. Hydrophobic polycation coatings on the surface of the substrates showed strong binding between the surfaces and the hydrophobic spike proteins of the influenza viruses through hydrophobic and electrostatic interactions, which causes conformational changes to the proteins, leading to disintegration of the viruses and the subsequent leakage of RNA [14]. Thus, positively charged surfaces with hydrophobicity appear to be an ideal characteristic to develop antiviral surfaces [15]. Yet another simplistic approach involves the use of naturally toxic materials to pathogens such as copper and silver $[4,9,10,13]$. However, for the commonly used metallic materials such as stainless steel and aluminum, there is still a necessity to identify the appropriate processing techniques to produce antiviral surfaces.

\subsection{Antibacterial surfaces}

Antibacterial surfaces are achieved by enhancing the adhesion resistance of bacteria to the surfaces (antifouling) and/or by destructing the bacteria by cell stretching and subsequent rupture (bactericidal) [5]. Antifouling characteristics are commonly influenced by surface chemistry, wettability, topography, and roughness. Besides, bacteria cell 
characteristics such as shape, size, hydrophobicity, gram stain of the cell, and extracellular polymeric substance (EPS) production influence the adhesion behavior of the bacteria with the contact surface $[5,16]$.

Similar to viruses, surface coating is one of the most frequent techniques to achieve antifouling (e.g. hydroxyapatite, titanium coatings) or bactericidal (e.g. silver, fluoride ion, antibiotics) characteristics, through modifying the surface chemistry [7]. Coatings are generally limited by nonuniformity, poor adhesive strength, and long-term instability. Surface topography modification is a physical approach to enhance adhesion resistance and the ability of the surface to kill bacteria, by tailoring wettability, roughness, topographical features, and surface chemistry. Several studies highlight that hierarchical microstructures with micro/nanofeatures smaller than the size of the bacteria provide superior antibacterial resistance for different bacteria types [17,18]. Lithography-based (UV, electron beam, nanoimprint, laser interference) and deposition-based (physical and chemical vapor deposition) techniques, plasma etching, anodization, and hydrothermal synthesis are some of the methods that can achieve fine nanostructures [1921]. The common drawbacks for most of these techniques are one or more of the following: expensive production, system complexity, mechanical and/or thermal instability of the produced surfaces, and difficult implementation on real 3D surface geometries.

Laser processing is a flexible, scalable, and chemical-free method that has the potential to overcome the current limitations. The development of ultrafast lasers demonstrates great potential to fabricate fine micro/nanoscale features on a wide variety of materials due to low ablation thresholds, plasma shielding effects, and minimized heat-affected zone and thermal stress, generated with pulses of extremely short duration (typically less than 10 picoseconds) [22,23].

\section{Laser surface texturing (LST):}

\subsection{LST for antibacterial surfaces}

Laser surface texturing using ultrashort laser pulses has been widely demonstrated to improve the performance of components in various applications. LST is used to create functional surfaces by altering characteristics such as friction and wear, wettability, biocompatibility, and optical response [24]. An interesting phenomenon more commonly observed with femtosecond $(f s)$ laser processing is the formation of laser-induced periodic surface structures (LIPSS) or ripples, in the scale of sub-wavelength periodicity, during ultrashort pulse irradiation with laser fluence near the ablation threshold [23].

(a)

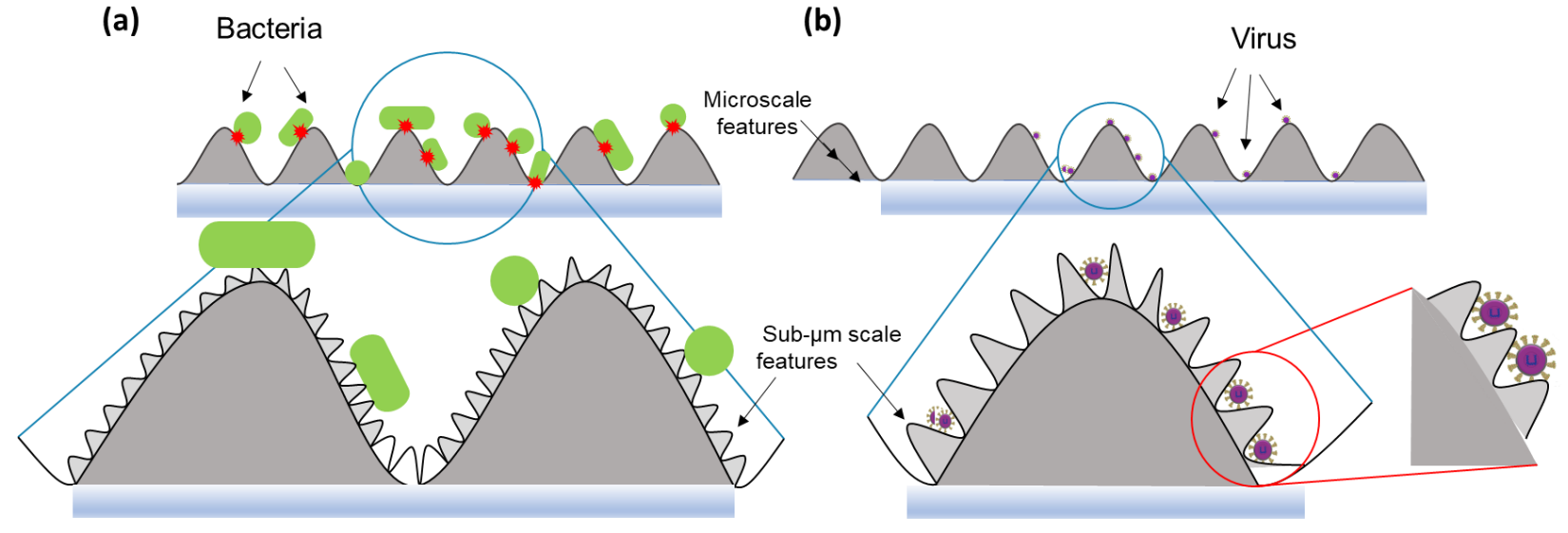

Figure 1. Schematic representation of the interaction between surfaces typically fabricated using LST and microorganisms: a) bacteria and b) virus [adapted with permission from [25]. Copyright (2020) American Chemical Society]

The ability of LST to achieve antibacterial behavior through fabricating fine surface features has already been demonstrated for several materials including titanium, steel, and gold [24,26-32]. LST using $f s$ lasers could achieve favorable antibacterial surface characteristics by tailoring the required surface properties such as surface chemistry, wettability, and topography $[21,24,26]$.

Surface topography is typically defined by the size, shape, density, and aspect ratio of the features [5]. The attachment point theory [33] suggests that there will be a stronger attachment if the surface feature size is larger than the organisms, due to the increase in the contact area. While microscale cavities typically enhance the adhesion of bacteria via increased contact area, bacterial adhesion occurs only at the tip of nanoscale features such as LIPSS (as seen in 
Fig. 1a), thus inhibiting further bacterial colonization. Cunha et al. [26] observed a reduction in Staphylococcus aureus (S. aureus) retention and biofilm formation on laser textured surfaces $\left(\mathrm{R}_{\mathrm{a}} \sim 0.3 \mu \mathrm{m}\right)$ due to the inhibition of bacteria adhesion by the dense submicron scale features (spacing $\sim 700 \mathrm{~nm}$, depth $\sim 200 \mathrm{~nm}$ ) which are smaller than the size of the bacteria. Contrastingly, Epperlein et al. [27] observed a complete biofilm formation over LIPSS surface for the same bacteria, S. aureus, on steel samples but a reduced bacteria adhesion for Escherichia coli (E. coli). A similar colonization of S. aureus on hierarchical micro/nanostructures was reported [28,31] on femtosecond laser textured Ti, but Pseudomonas aeruginosa ( $P$. aeruginosa) showed better resistance against bacteria adhesion. The difference in this behavior between the different bacteria strains with varying shapes was attributed to the propensity of bacteria to orient themselves to the structures in order to increase the contact area and achieve EPS formation [27,28,31]. Regarding the effect of surface roughness, there exists an optimal roughness to reduce the bacteria retention as both the mirror-finish surfaces and extremely rough surfaces have shown increased adhesion and biofilm formation [26].

Surface wettability can be controlled by modifying the surface chemistry and roughness. According to Cassie-Baxter's wettability model [34] for heterogenous rough surfaces, the formation of air bubbles between the solid surface and the liquid phase causes the droplet to be in contact only at the top of the microgrooves; which can be exploited to reduce the bacteria contact with the substrate [21]. Typically, hydrophobicity is shown to improve the antifouling characteristics of many materials [24,32]. However, a contradicting trend of reduced bacterial adhesion for laser textured surfaces with increased hydrophilicity [Contact angle $(\mathrm{CA}) \sim 13-32^{\circ}$ ] has been reported [26]. It can be noted from the literature that the influences of surface wettability, topography, and roughness on bacterial adhesion are complex and interconnected. Lutey et al. [35] attempted to distinguish the effects of wettability, surface morphology, and topography on the antifouling effects and observed that: i) bacteria strain (and hence cell geometry) was more influential than the topography, with E.coli showing better adhesion resistance than $S$. aureus, in confirmation with the previous results [27,28], ii) the surface with finer topography (LIPSS) produced better resistance against bacteria, irrespective of the bacteria type, iii) hydrophobic conditions performed better than the hydrophilic samples for the same LIPSS morphology, iv) surface features with size larger than that of bacteria had higher bacteria retention, despite the superhydrophobic condition; highlighting the dominant effect of surface topography over wettability.

\subsection{LST for antiviral surfaces}

Despite the proven ability of laser surface texturing in obtaining antibacterial surfaces, its applicability to the development of antiviral surfaces is yet to be explored. Recently, Siddhiquie et al. [36] reviewed the potential of nanoparticle coating and surface texturing techniques to achieve antiviral surface characteristics. Antiviral coatings [37], especially fullerene $\left(\mathrm{C}_{60}\right)$ nanoparticle coatings, is identified as a better technique to achieve antiviral surfaces as it results in deterioration of the outer lipid layer through lipid peroxidation, causing resistance against the virus [38].

Very recently, Hasan et al. [39] reported on the effect of surface nanostructures on the antiviral characteristics for the first time. Wet etching was used to fabricate nanostructures of $23 \mathrm{~nm}$ width randomly spread over parallel ridges of $161 \mathrm{~nm}$ on aluminum 6063 alloy surface. In addition to their resistance against bacteria (S. aureus and P. aeruginosa), these nanostructures were effective against nonenveloped [rhinovirus (RV)] and enveloped viruses [respiratory syncytial virus (RSV)]. The viability of both RV and RSV viruses significantly reduced upon the exposure of $24 \mathrm{~h}$ to the nanostructure surfaces. The same research group extended a similar study to SARS-CoV-2 [40] and found that the viability of the virus significantly reduced within $6 \mathrm{~h}$ of exposure to the etched nanostructured surface, in comparison to $24 \mathrm{~h}$ exposure needed to attain the same effect on the smooth surface. One of the possible mechanisms postulated is the accelerated rupturing of the lipid envelope by the nanostructures; which is disputed as the nanostructured surface shows even higher resistance against nonenveloped RV virus. The mechanisms behind the interaction of the virus with the nanostructures are yet to be explored. It has to be noted that the surface of the etched substrate was nonuniform (with a mean roughness of $1 \mu \mathrm{m}$ ) with large structures where virus particles could possibly get trapped $[39,40]$. In this study [39], nanostructure formation modified the initially hydrophobic (CA - 96.3 $)$ surface to highly hydrophilic $\left(\mathrm{CA}-17.7^{\circ}\right)$, possibly due to the surface chemistry alteration (oxidation) by etching. It is perceived that the influence of surface topography can still be effective in antiviral resistance despite the unfavorable wettability and poor surface finish.

In order to reduce the virus adhesion and/or to pierce the outer wall of the virus envelope, the surface topography should have features smaller than the size of the virus particles (ranging from $20 \mathrm{~nm}$ to $300 \mathrm{~nm}$ generally) [13]. LST can achieve multiscale features with sub- $\mu \mathrm{m}$ LIPSS features but the size and spacing of these features are limited primarily by the laser wavelength. For metallic surfaces in focus here, the typical minimum spatial resolution of the LIPSS features produced by LST is higher $(>300 \mathrm{~nm})$ than the required dimensions $[21,26,28,35]$. However, for a 
surface with features larger than the size of the virus, there is a possibility of increased virus adsorption in the cavities between the peaks (as illustrated in Fig. 1b), like the increased surface interaction with the bacteria [21].

As hydrophobic surfaces are shown to be effective in reducing the hydrogen bonding and hence virus-surface adsorption and retention [9], increasing the hydrophobicity of the surfaces by LST can be considered as a possibility to achieve antiviral surfaces. Nevertheless, previous research on antifouling studies evidently demonstrated that the beneficial influence of hydrophobicity is effective only when the feature size is smaller than the size of the bacteria $[5,26,28]$. Interestingly, Truong et al. [18] observed that the formation of air bubbles in the microscale cavities prevents bacterial adhesion to the substrate surface even though the size of the cavities is larger than that of the bacteria. However, the air bubbles are reported to disappear within an hour followed by increased adhesion, indicating the limitation of LST using $f_{s}$ lasers for fabricating the required surfaces for the virus.
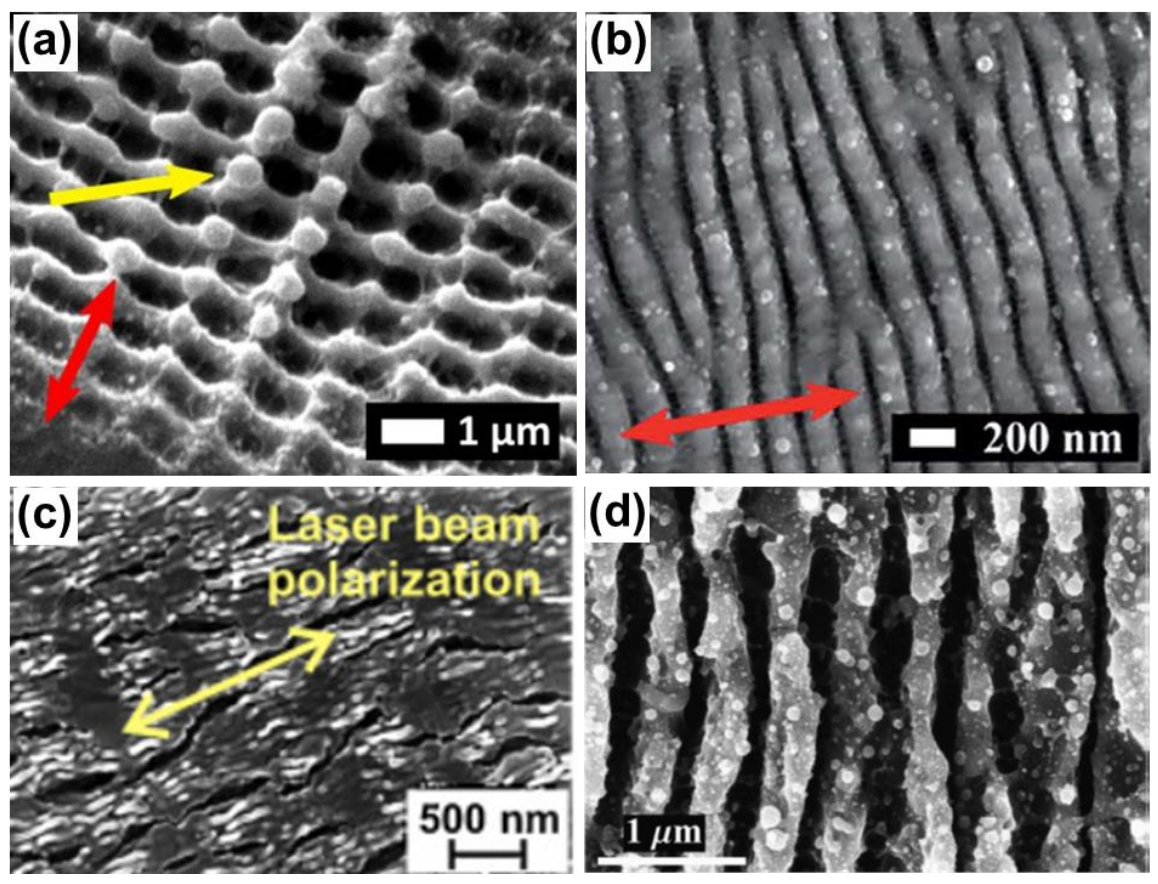

Figure 2. Fabrication of nanostructures using femtosecond laser processing: a) DLIP with LIPSS features on stainless samples (yellow arrows represent nanoscale protrusions) [41] (reprinted with permission), b) LIPSS features on stainless steel produced by $257 \mathrm{~nm}$ wavelength $f_{s}$ laser pulses [42] - Published by The Royal Society of Chemistry, c) High spatial frequency LIPSS (HSFL) features with sub-100 nm periodicity features on titanium [43]

(reprinted with permission), and d) LIPSS covered with nanoscale features on titanium [44] (reprinted with permission)

Despite the limitations plaguing the application of $f s$-LST for antiviral surfaces, ultrafast lasers offer several new possibilities to obtain nanostructures.

- Recently, Direct Laser Interference Patterning (DLIP), a technique that uses periodic interference patterns created by overlapping multiple coherent beams to create periodic sub- $\mu \mathrm{m}$ structures, has been employed for antifouling applications [45]. DLIP on stainless steel samples produced periodic holes and cones of $850 \mathrm{~nm}$ periodicity which demonstrated a significant reduction in E. coli and $S$. aureus adhesion. DLIP provides greater flexibility, especially with ultrashort pulses, to produce unique microstructures (as seen in Fig. 2a) due to the interaction between LIPSS and DLIP structures, both of which can be controlled independently $[41,46]$. Nevertheless, the limitation on the feature size still depends upon the wavelength of the beam.

- Femtosecond laser processing using shorter (UV) wavelengths has been used for dielectrics [47] where LIPSS features of less than $100 \mathrm{~nm}$ periodicity were demonstrated for different diamond materials. Fotis et al. [42] expanded this approach for metals to produce LIPSS with ripples width as small as $76 \mathrm{~nm}$, as shown in Fig. 2b, on stainless steel using a $257 \mathrm{~nm} f$ s laser beam.

- Beam shaping techniques such as double-pulse irradiation, using time delays between two $f s$ pulses and crossed polarization, can be used to produce complex LIPSS structures [24]. With this technique, Bonse et 
al. [48] reported on the feasibility to create LIPSS features with periodicity less than $100 \mathrm{~nm}$ for titanium (Fig. 2c), which is later employed for different metals [49,50].

- In addition to the periodic LIPSS structures, $f_{s}$ laser processing can produce different nanostructures including nanopores, nanoprotrusions, nanospheres, nanowires, nanorims of dimensions smaller than $50 \mathrm{~nm}$ on metals [23,44,51], which can also be overlapped with LIPSS to produce hierarchical structures as seen in Fig. 2d [44]. Non-uniform nanostructures can be helpful to prevent the propensity of microorganisms to orient along the regular structures, such as grooves, to increase their contact area with the surfaces [52].

Despite the current lack of literature on the fabrication of antiviral surfaces by LST, these promising results underline the possibility of using ultrafast lasers in achieving an ideal surface topography with favorable wettability (superhydrophobic), feature size (smaller than the virus size), and feature shape (that promotes adhesion resistance) to realize antiviral surfaces - that effectively resist, repel and kill the virus particles. Also, laser processing has been used to enhance the adhesive strength of coatings in multiple applications [53,54]. This hybrid approach can also be adapted to improve the mechanical strength and long-term stability of superhydrophobic coatings typically used for producing antiviral surfaces.

\section{Summary:}

The objective of this paper was to examine the possibilities of exploiting laser-based manufacturing processes, particularly, laser surface texturing, for fabricating antiviral surfaces which is capable to resist the adhesion of viruses and/or destruct them upon contact.

Based on the literature review, we observed that the current surface modification techniques for antiviral surfaces are limited mostly to surface coatings. To evaluate the applicability of LST for viruses, we first examined the LST of antibacterial surfaces fabricated using LST, which is well-documented in the literature. Several studies highlight that hierarchical microstructures with micro/nanofeatures effectively smaller than the size of the bacteria provide superior resistance against different bacteria types. However, to replicate a similar behavior for viruses, surface topography should possess features smaller than the size of the virus (less than $300 \mathrm{~nm}$ ). LST can achieve multiscale topography with fine LIPSS features but the periodicity of the LIPSS depends on the wavelength; limiting the ability to fabricate fine nanoscale features less than $100 \mathrm{~nm}$.

Despite the current limitations with LST, the recent developments in ultrafast laser processing display great promise to achieve fine nanostructures through different strategies: a) Direct Laser Interference Patterning (DLIP) with LIPSS, b) femtosecond laser irradiation using UV wavelength, c) beam shaping techniques and, d) fabrication of non-uniform nanostructures (size smaller than $50 \mathrm{~nm}$ ) over LIPSS patterns. The adoption of these new techniques could advance the ability of LST to obtain the fine nanoscale resolution required for antiviral surfaces.

\section{References:}

[1] Rawlinson, S., Ciric, L., and Cloutman-Green, E., 2020, "COVID-19 Pandemic - Let's Not Forget Surfaces," J. Hosp. Infect., 105(4), pp. 790-791.

[2] Van Doremalen, N., Bushmaker, T., Morris, D. H., Holbrook, M. G., Gamble, A., Williamson, B. N., Tamin, A., Harcourt, J. L., Thornburg, N. J., and Gerber, S. I., 2020, "Aerosol and Surface Stability of SARS-CoV-2 as Compared with SARS-CoV-1,” N. Engl. J. Med., 382(16), pp. 1564-1567.

[3] Kampf, G., Todt, D., Pfaender, S., and Steinmann, E., 2020, "Persistence of Coronaviruses on Inanimate Surfaces and Their Inactivation with Biocidal Agents," J. Hosp. Infect., 104(3), pp. 246-251.

[4] Bueckert, M., Gupta, R., Gupta, A., Garg, M., and Mazumder, A., 2020, "Infectivity of SARS-CoV-2 and Other Coronaviruses on Dry Surfaces: Potential for Indirect Transmission," Materials (Basel)., 13(22), p. 5211.

[5] Linklater, D. P., Baulin, V. A., Juodkazis, S., Crawford, R. J., Stoodley, P., and Ivanova, E. P., 2020, "Mechano-Bactericidal Actions of Nanostructured Surfaces," Nat. Rev. Microbiol., 19(1), pp. 8-22.

[6] Genzer, J., and Efimenko, K., 2006, "Recent Developments in Superhydrophobic Surfaces and Their Relevance to Marine Fouling: A Review," Biofouling, 22(5), pp. 339-360.

[7] Hasan, J., Crawford, R. J., and Ivanova, E. P., 2013, “Antibacterial Surfaces: The Quest for a New Generation of Biomaterials," Trends Biotechnol., 31(5), pp. 295-304.

[8] Otter, J., Brophy, K., Palmer, J., Harrison, N., Riley, J., Williams, D., and Larrouy-Maumus, G., 2020, "Smart Surfaces to Tackle Infection and Antimicrobial Resistance," Briefing paper, Imperial College London.

[9] Joonaki, E., Hassanpouryouzband, A., Heldt, C. L., and Areo, O., 2020, "Surface Chemistry Can Unlock Drivers of Surface Stability of SARS-CoV-2 in a Variety of Environmental Conditions," Chem, 6(9), pp. 
2135-2146.

[10] Xue, X., Ball, J. K., Alexander, C., and Alexander, M. R., 2020, "All Surfaces Are Not Equal in Contact Transmission of SARS-CoV-2," Matter, 3(5), pp. 1433-1441.

[11] Chin, A. W. H., Chu, J. T. S., Perera, M. R. A., Hui, K. P. Y., Yen, H.-L., Chan, M. C. W., Peiris, M., and Poon, L. L. M., 2020, "Stability of SARS-CoV-2 in Different Environmental Conditions," The Lancet Microbe, 1(1), p. e10.

[12] Casanova, L. M., Jeon, S., Rutala, W. A., Weber, D. J., and Sobsey, M. D., 2010, "Effects of Air Temperature and Relative Humidity on Coronavirus Survival on Surfaces," Appl. Environ. Microbiol., 76(9), pp. 27122717.

[13] Sun, Z., and Ostrikov, K. (Ken), 2020, "Future Antiviral Surfaces: Lessons from COVID-19 Pandemic," Sustain. Mater. Technol., 25, p. e00203.

[14] Hsu, B. B., Wong, S. Y., Hammond, P. T., Chen, J., and Klibanov, A. M., 2011, "Mechanism of Inactivation of Influenza Viruses by Immobilized Hydrophobic Polycations,” Proc. Natl. Acad. Sci., 108(1), pp. 61-66.

[15] Pandey, L. M., 2020, "Surface Engineering of Personal Protective Equipments (PPEs) to Prevent the Contagious Infections of SARS-CoV-2," Surf. Eng., 36(9), pp. 901-907.

[16] Majhi, S., and Mishra, A., 2020, "Modulating Surface Energy and Surface Roughness for Inhibiting Microbial Growth BT - Engineered Antimicrobial Surfaces," S. Snigdha, S. Thomas, E.K. Radhakrishnan, and N. Kalarikkal, eds., Springer Singapore, Singapore, pp. 109-121.

[17] Helbig, R., Günther, D., Friedrichs, J., Rößler, F., Lasagni, A., and Werner, C., 2016, “The Impact of Structure Dimensions on Initial Bacterial Adhesion," Biomater. Sci., 4(7), pp. 1074-1078.

[18] Truong, V. K., Webb, H. K., Fadeeva, E., Chichkov, B. N., Wu, A. H. F., Lamb, R., Wang, J. Y., Crawford, R. J., and Ivanova, E. P., 2012, "Air-Directed Attachment of Coccoid Bacteria to the Surface of Superhydrophobic Lotus-like Titanium,” Biofouling, 28(6), pp. 539-550.

[19] Tripathy, A., Sen, P., Su, B., and Briscoe, W. H., 2017, "Natural and Bioinspired Nanostructured Bactericidal Surfaces," Adv. Colloid Interface Sci., 248, pp. 85-104.

[20] Coblas, D. G., Fatu, A., Maoui, A., and Hajjam, M., 2015, "Manufacturing Textured Surfaces: State of Art and Recent Developments," Proc. Inst. Mech. Eng. Part J J. Eng. Tribol., 229(1), pp. 3-29.

[21] Ijaola, A. O., Bamidele, E. A., Akisin, C. J., Bello, I. T., Oyatobo, A. T., Abdulkareem, A., Farayibi, P. K., and Asmatulu, E., 2020, "Wettability Transition for Laser Textured Surfaces: A Comprehensive Review," Surfaces and Interfaces, 21, p. 100802.

[22] Phillips, K. C., Gandhi, H. H., Mazur, E., and Sundaram, S. K., 2015, "Ultrafast Laser Processing of Materials: A Review," Adv. Opt. Photonics, 7(4), pp. 684-712.

[23] Vorobyev, A. Y., and Guo, C., 2013, "Direct Femtosecond Laser Surface Nano/Microstructuring and Its Applications," Laser Photon. Rev., 7(3), pp. 385-407.

[24] Stratakis, E., Bonse, J., Heitz, J., Siegel, J., Tsibidis, G. D., Skoulas, E., Papadopoulos, A., Mimidis, A., Joel, A. C., Comanns, P., Krüger, J., Florian, C., Fuentes-Edfuf, Y., Solis, J., and Baumgartner, W., 2020, "Laser Engineering of Biomimetic Surfaces,” Mater. Sci. Eng. R Reports, 141, p. 100562.

[25] Siddiquie, R. Y., Gaddam, A., Agrawal, A., Dimov, S. S., and Joshi, S. S., 2020, "Anti-Biofouling Properties of Femtosecond Laser-Induced Submicron Topographies on Elastomeric Surfaces," Langmuir, 36(19), pp. $5349-5358$.

[26] Cunha, A., Elie, A.-M., Plawinski, L., Serro, A. P., Botelho do Rego, A. M., Almeida, A., Urdaci, M. C., Durrieu, M.-C., and Vilar, R., 2016, "Femtosecond Laser Surface Texturing of Titanium as a Method to Reduce the Adhesion of Staphylococcus Aureus and Biofilm Formation," Appl. Surf. Sci., 360, pp. 485-493.

[27] Epperlein, N., Menzel, F., Schwibbert, K., Koter, R., Bonse, J., Sameith, J., Krüger, J., and Toepel, J., 2017, "Influence of Femtosecond Laser Produced Nanostructures on Biofilm Growth on Steel," Appl. Surf. Sci., 418, pp. 420-424.

[28] Fadeeva, E., Truong, V. K., Stiesch, M., Chichkov, B. N., Crawford, R. J., Wang, J., and Ivanova, E. P., 2011, "Bacterial Retention on Superhydrophobic Titanium Surfaces Fabricated by Femtosecond Laser Ablation," Langmuir, 27(6), pp. 3012-3019.

[29] Jalil, S. A., Akram, M., Bhat, J. A., Hayes, J. J., Singh, S. C., ElKabbash, M., and Guo, C., 2020, “Creating Superhydrophobic and Antibacterial Surfaces on Gold by Femtosecond Laser Pulses," Appl. Surf. Sci., 506, p. 144952.

[30] Villapún, V. M., Gomez, A. P., Wei, W., Dover, L. G., Thompson, J. R., Barthels, T., Rodriguez, J., Cox, S., and González, S., 2020, "Development of Antibacterial Steel Surfaces through Laser Texturing," APL Mater., 8(9), p. 091108.

[31] Shaikh, S., Kedia, S., Singh, D., Subramanian, M., and Sinha, S., 2018, "Surface Texturing of Ti6A14V Alloy 
Using Femtosecond Laser for Superior Antibacterial Performance,” arXiv, 31(2), p. 022011.

[32] Pan, Q., Cao, Y., Xue, W., Zhu, D., and Liu, W., 2019, "Picosecond Laser-Textured Stainless Steel Superhydrophobic Surface with an Antibacterial Adhesion Property,” Langmuir, 35(35), pp. 11414-11421.

[33] Scardino, A. J., Guenther, J., and de Nys, R., 2008, "Attachment Point Theory Revisited: The Fouling Response to a Microtextured Matrix," Biofouling, 24(1), pp. 45-53.

[34] Cassie, A. B. D., and Baxter, S., 1944, "Wettability of Porous Surfaces," Trans. Faraday Soc., 40, pp. 546551.

[35] Lutey, A. H. A., Gemini, L., Romoli, L., Lazzini, G., Fuso, F., Faucon, M., and Kling, R., 2018, “Towards Laser-Textured Antibacterial Surfaces," Sci. Rep., 8(1), pp. 1-10.

[36] Siddiquie, R. Y., Agrawal, A., and Joshi, S. S., 2020, "Surface Alterations to Impart Antiviral Properties to Combat COVID-19 Transmission,” Trans. Indian Natl. Acad. Eng., pp. 1-5.

[37] Ruiz-Hitzky, E., Darder, M., Wicklein, B., Ruiz-Garcia, C., Martín-Sampedro, R., Del Real, G., and Aranda, P., 2020, "Nanotechnology Responses to COVID-19," Adv. Healthc. Mater., 9(19), p. 2000979.

[38] Sayes, C. M., Gobin, A. M., Ausman, K. D., Mendez, J., West, J. L., and Colvin, V. L., 2005, “Nano-C60 Cytotoxicity Is Due to Lipid Peroxidation," Biomaterials, 26(36), pp. 7587-7595.

[39] Hasan, J., Xu, Y., Yarlagadda, T., Schuetz, M., Spann, K., and Yarlagadda, P. K. D. V, 2020, “Antiviral and Antibacterial Nanostructured Surfaces with Excellent Mechanical Properties for Hospital Applications," ACS Biomater. Sci. Eng., 6(6), pp. 3608-3618.

[40] Hasan, J., Pyke, A., Nair, N., Yarlagadda, T., Will, G., Spann, K., and Yarlagadda, P. K. D. V., 2020, "Antiviral Nanostructured Surfaces Reduce the Viability of SARS-CoV-2," ACS Biomater. Sci. Eng., 6(9), pp. $4858-4861$.

[41] Alamri, S., Fraggelakis, F., Kunze, T., Krupop, B., Mincuzzi, G., Kling, R., and Lasagni, A. F., 2019, “On the Interplay of DLIP and LIPSS upon Ultra-Short Laser Pulse Irradiation,” Materials (Basel)., 12(7), p. 1018.

[42] Fraggelakis, F., Mincuzzi, G., Manek-Hönninger, I., Lopez, J., and Kling, R., 2018, "Generation of Microand Nano-Morphologies on a Stainless Steel Surface Irradiated with 257 Nm Femtosecond Laser Pulses,” RSC Adv., 8(29), pp. 16082-16087.

[43] Bonse, J., Höhm, S., Koter, R., Hartelt, M., Spaltmann, D., Pentzien, S., Rosenfeld, A., and Krüger, J., 2016, "Tribological Performance of Sub-100-Nm Femtosecond Laser-Induced Periodic Surface Structures on Titanium," Appl. Surf. Sci., 374, pp. 190-196.

[44] Vorobyev, A. Y., and Guo, C., 2007, "Femtosecond Laser Structuring of Titanium Implants,” Appl. Surf. Sci., 253(17), pp. 7272-7280.

[45] Peter, A., Lutey, A. H. A., Faas, S., Romoli, L., Onuseit, V., and Graf, T., 2020, "Direct Laser Interference Patterning of Stainless Steel by Ultrashort Pulses for Antibacterial Surfaces," Opt. Laser Technol., 123, p. 105954.

[46] Müller, D. W., Fox, T., Grützmacher, P. G., Suarez, S., and Mücklich, F., 2020, “Applying Ultrashort Pulsed Direct Laser Interference Patterning for Functional Surfaces," Sci. Rep., 10(1), pp. 1-14.

[47] Trucchi, D. M., Bellucci, A., Girolami, M., Mastellone, M., and Orlando, S., 2017, "Surface Texturing of CVD Diamond Assisted by Ultrashort Laser Pulses," Coatings, 7(11), p. 185.

[48] Bonse, J., Krüger, J., Höhm, S., and Rosenfeld, A., 2012, "Femtosecond Laser-Induced Periodic Surface Structures," J. Laser Appl., 24(4), p. 42006.

[49] Fraggelakis, F., Mincuzzi, G., Lopez, J., Manek-Hönninger, I., and Kling, R., 2019, “Controlling 2D Laser Nano Structuring over Large Area with Double Femtosecond Pulses,” Appl. Surf. Sci., 470, pp. 677-686.

[50] Jalil, S. A., Yang, J., Elkabbash, M., Cong, C., and Guo, C., 2019, "Formation of Controllable 1D and 2D Periodic Surface Structures on Cobalt by Femtosecond Double Pulse Laser Irradiation,” Appl. Phys. Lett., 115(3), p. 031601.

[51] Ahmmed, K. M., Grambow, C., and Kietzig, A.-M., 2014, "Fabrication of Micro/Nano Structures on Metals by Femtosecond Laser Micromachining," Micromachines, 5(4), pp. 1219-1253.

[52] Luo, X., Yao, S., Zhang, H., Cai, M., Liu, W., Pan, R., Chen, C., Wang, X., Wang, L., and Zhong, M., 2020, "Biocompatible Nano-Ripples Structured Surfaces Induced by Femtosecond Laser to Rebel Bacterial Colonization and Biofilm Formation,” Opt. Laser Technol., 124, p. 105973.

[53] Arroyo, J. M., Diniz, A. E., and de Lima, M. S. F., 2010, “Cemented Carbide Surface Modifications Using Laser Treatment and Its Effects on Hard Coating Adhesion," Surf. Coatings Technol., 204(15), pp. 24102416.

[54] Kromer, R., Costil, S., Verdy, C., Gojon, S., and Liao, H., 2018, "Laser Surface Texturing to Enhance Adhesion Bond Strength of Spray Coatings - Cold Spraying, Wire-Arc Spraying, and Atmospheric Plasma Spraying," Surf. Coatings Technol., 352, pp. 642-653. 